\title{
Distinct Matroid Base Weights and Additive Theory
}

\author{
Y. O. Hamidoune* $\quad$ I.P. da Silva ${ }^{\dagger}$
}

\begin{abstract}
Let $M$ be a matroid on a set $E$ and let $w: E \longrightarrow G$ be a weight function, where $G$ is a cyclic group. Assuming that $w(E)$ satisfies the Pollard's Condition (i.e. Every non-zero element of $w(E)-w(E)$ generates $G)$, we obtain a formulae for the number of distinct base weights. If $|G|$ is a prime, our result coincides with a result Schrijver and Seymour.

We also describe Equality cases in this formulae. In the prime case, our result generalizes Vosper's Theorem.
\end{abstract}

\section{Introduction}

Let $G$ be a finite cyclic group and let $A, B$ be nonempty subsets of $G$. The starting point of Minkowski set sum estimation is the inequality $|A+B| \geq \min (|G|,|A|+|B|-1)$, where $|G|$ is a prime, proved by Cauchy [2] and rediscovered by Davenport [4]. The first generalization of this result, due to Chowla [3], states that $|A+B| \geq \min (|G|,|A|+|B|-1)$, if there is a $b \in B$ such that every non-zero element of $B-b$ generates $G$. In order to generalize his extension of the Cauchy-Davenport Theorem [1] to composite moduli, Pollard introduced in [12] the following more sophisticated Chowla type condition: Every non-zero element of $B-B$ generates $G$.

Equality cases of the Cauchy-Davenport were determined by Vosper in [16, 17]. Vosper's Theorem was generalized by Kemperman [9]. We need only a light form of Kemperman's result stated in the beginning of Kemperman's paper.

We need the following combination of Chowla and Kemperman results:

Theorem A (Chowla [3], Kemperman [9]) Let $A, B$ be non-empty subsets of a cyclic group $G$ with $|A|,|B| \geq 2$ such that for some $b \in B$, every non-zero element of $B-b$ generates $G$. Then $|A+B| \geq|A|+|B|-1$.

Moreover $|A+B|=|A|+|B|-1$ if and only if $A+B$ is an arithmetic progression.

A shortly proved generalization of this result to non-abelian groups is obtained in [8].

${ }^{*}$ Université Pierre et Marie Curie, E. Combinatoire, Case 189, 4 Place Jussieu, 75005 Paris, France. yha@ccr.jussieu.fr

${ }^{\dagger}$ CELC/Universidade de Lisboa, Faculdade de Ciências, Campo Grande, edifício C6 - Piso 2, 1749-016 Lisboa, Portugal.isilva@cii.fc.ul.pt 
Zero-sum problems form another developing area in Additive Combinatorics having several applications. The Erdös-Ginzburg-Ziv Theorem [6] was the starting point of this area. This result states that a sequence of elements of an abelian group $G$ with length $\geq 2|G|-1$ contains a zero-sum subsequence of length $=|G|$.

The reader may find some details on these two areas of Additive Combinatorics in the text books: Nathanson [10], Geroldinger-Halter-Koch [7] and Tao-Vu [15]. More specific questions may be found in Caro's survey paper [1].

The notion of a matroid was introduced by Whitney in 1935 as a generalization of a matrix. Two pioneer works connecting matroids and Additive Combinatorics are due to SchrijverSeymour [14, Dias da Silva-Nathanson [5]. Recently, in [13, orientability of matroids is naturally related with an open problem on Bernoulli matrices.

Stating the first result requires some vocabulary:

Let $E$ be a finite set. The set of the subsets of $E$ will be denoted by $2^{E}$.

A matroid over $E$ is an ordered pair $(E, \mathcal{B})$ where $\mathcal{B} \subseteq 2^{E}$ satisfies the following axioms:

(B1) $\mathcal{B} \neq \emptyset$.

(B2) For all $B, B^{\prime} \in \mathcal{B}$, if $B \subseteq B^{\prime}$ then $B=B^{\prime}$.

(B3) For all $B, B^{\prime} \in \mathcal{B}$ and $x \in B \backslash B^{\prime}$, there is a $y \in B^{\prime} \backslash B$ such that $(B \backslash\{x\}) \cup\{y\} \in \mathcal{B}$.

A set belonging to $\mathcal{B}$ is called a basis of the matroid $M$.

The rank of a subset $A \subseteq E$ is by definition $r_{M}(A):=\max \{|B \cap A|: B$ is a basis of $M\}$. We write $r(M)=r(E)$. The reference to $M$ could be omitted. A hyperplane of the matroid $M$ is a maximal subset of $E$ with $\operatorname{rank}=r(M)-1$.

The uniform matroid of rank $r$ on a set $E$ is by definition $\mathcal{U}_{r}(E)=\left(E,\left(\begin{array}{l}E \\ r\end{array}\right)\right)$, where $\left(\begin{array}{l}E \\ r\end{array}\right)$ is the set of all $r$-subsets of $E$. Let $M$ be a matroid on $E$ and let $N$ be a matroid on $F$. We define the direct sum:

$M \oplus N=(E \times\{0\} \cup F \times\{1\},\{B \times\{0\} \cup C \times\{1\}: B$ is a base of $M$ and $C$ is a base of $N\}$.

Let $w: E \longrightarrow G$ be a weight function, where $G$ is an abelian group. The weight of a subset $X$ is by definition

$$
X^{w}=\sum_{x \in X} w(x)
$$

The set of distinct base weights is

$$
M^{w}=\left\{B^{w}: B \text { is a basis of } M\right\} .
$$

Suppose now $|G|=p$ is a prime number. Schrijver and Seymour proved that $\left|M^{w}\right| \geq$ $\min \left(p, \sum_{g \in G} r\left(w^{-1}(g)\right)-r(M)+1\right)$. Let $A$ and $B$ be subsets of $G$. Define $w: A \times\{0\} \cup B \times\{1\}$, 
by the relation $w(x, y)=x$. Then

$$
\left(\mathcal{U}_{1}(A) \oplus \mathcal{U}_{1}(B)\right)^{w}=A+B
$$

Applying their result to this matroid, Schrijver and Seymour obtained the Cauchy-Davenport Theorem.

Let $x_{1}, \ldots, x_{2 p-1} \in G$. Consider the uniform matroid $M=\mathcal{U}_{p}(E)$, of rank $p$ over the set $E=\{1, \ldots, 2 p-1\}$, with weight function $w(i)=x_{i}$. In order to prove the Erdős-Ginzburg-Ziv Theorem [6], one may clearly assume that no element is repeated $p$ times. In particular for every $g \in G, r\left(w^{-1}(g)\right)=\left|w^{-1}(g)\right|$. Applying Schrijver and Seymour to this matroid we have:

$$
\left|M^{w}\right| \geq \min \left(|G|, \sum_{g \in G} r\left(w^{-1}(g)\right)-r(M)+1\right)=\min \left(p, \sum_{g \in G}\left|w^{-1}(g)\right|-p+1\right)=p .
$$

Thus Schrijver-Seymour result also implies the Erdős-Ginzburg-Ziv Theorem [6] in a prime order.

In the present work, we prove the following result:

Theorem 1 Let $G$ be a cyclic group, $M$ be a matroid on a finite set $E$ with $r(M) \geq 1$ and let $w: E \longrightarrow G$ be a weight function. Assume moreover that every non-zero element of $w(E)-w(E)$ generates $G$. Then

$$
\left|M^{w}\right| \geq \min \left(|G|, \sum_{g \in G} r\left(w^{-1}(g)\right)-r(M)+1\right),
$$

where $M^{w}$ denotes the set of distinct base weights. Moreover, if Equality holds in (1) then one of the following conditions holds:

(i) $r(M)=1$ or $M^{w}$ is an arithmetic progression.

(ii) There is a hyperplane $H$ of $M$ such that $M^{w}=g+(M / H)^{w}$, for some $g \in G$.

If $G$ has a prime order, then the condition on $w(E)-w(E)$ holds trivially. In this case (1) reduces to the result of Schrijver-Seymour.

\section{Terminology and Preliminaries}

Let $M$ be a matroid on a finite set $E$. One may see easily from the definitions that all bases a matroid have the same cardinality. A circuit of $M$ is a minimal set not contained in a base. A loop is an element $x$ such that $\{x\}$ is a circuit. By the definition bases contain no loop. The closure of a subset $A \subseteq E$ is by definition

$$
c l(A)=\{x \in A: r(A \cup x)=r(A)\} .
$$

Note that an element $x \in \operatorname{cl}(A)$ if and only if $x \in A$, or there is circuit $C$ such $x \in C$ and $C \backslash\{x\} \subseteq A$. 
Given a matroid $M$ on a set $E$ and a subset $A \subseteq E$. Then $\mathcal{B} / A:=\{J \backslash A: J$ is a basis of $M$ with $\mid B \cap$ $A \mid=r(A)\}$. One may see easily that $M / A=(E \backslash A, \mathcal{B} / A)$ is a matroid on $E \backslash A$. We say that this matroid is obtained from $M$ contracting $A$. Notice that $r_{M / A}(X)=r_{M}(X \cup A)-r_{M}(A)$.

Recall the following easy lemma:

Lemma 2 Let $M$ be a matroid on a finite set $E$ and let $U, V$ be disjoint subsets of $E$. Then

- $M / U$ and $M / \operatorname{cl}(U)$ have the same bases. In particular, $(M / U)^{w}=(M / \operatorname{cl}(U))^{w}$.

- $(M / U) / V=M /(U \cup V)$.

For more details on matroids, the reader may refer to one of the text books: Welsh [18] or White [19].

For $u \in E$, we put

$$
G_{u}:=\left\{g \in G: u \in \operatorname{cl}\left(w^{-1}(g)\right)\right\}
$$

We recall the following lemma proved by Schrijver and Seymour in [14]:

Lemma B Let $M$ be a matroid on a finite set $E$ and let $w: E \longrightarrow G$ be a weight function. Then for every non-loop element $u \in E$,

$$
(M / u)^{w}+G_{u} \subseteq M^{w} .
$$

Proof. Take a basis $B$ of $M / u$ and an element $g \in G_{u}$. If $g=w(u)$ then, by definition of contraction, $B \cup\{u\}$ is a basis of $M$ and $B^{w}+w(u) \in M^{w}$. If $g \neq w(u)$, there is a circuit $C$ containing $u$ such that $\emptyset \neq C \backslash\{u\} \subseteq w^{-1}(g)$. For some $v \in C \backslash\{u\}$ the subset $B \cup\{v\}$ must be a basis of $M$ otherwise $C \backslash\{v\} \subseteq \operatorname{cl}(B)$, implying that $u \in \operatorname{cl}(B)$, in contradiction with the assumption that $B$ is a basis of $M / u$. Therefore $(B \cup\{v\})^{w}=B^{w}+g \in M^{w}$.

\section{Proof of the main result}

We shall now prove our result:

Proof of Theorem 1:

We first prove (11) by induction on the rank of $M$. The result holds trivially if $r(M)=1$. Since $r(M) \geq 1, M$ contains a non-loop element. Take an arbitrary non-loop element $y$.

$$
\begin{aligned}
\left|M^{w}\right| & \geq\left|(M / y)^{w}+G_{y}\right| \\
& \geq\left|(M / y)^{w}\right|+\left|G_{y}\right|-1 \\
& \geq \sum_{g \in G} r\left(w^{-1}(g)\right)-r(M)+1
\end{aligned}
$$


The first inequality follows from Lemma $\mathrm{B}$, the second follows by Theorem $\mathrm{A}$ and the third is a direct consequence of the definitions of $M / u$ and $G_{u}$. This proves the first part of the theorem.

Suppose now that Equality holds in (1) and that Condition (i) is not satisfied. In particular $r(M) \geq 2$. Also $\left|M^{w}\right| \geq 2$, otherwise $M^{w}$ is a progression, a contradiction.

We claim that there exits a non-loop element $u \in E$ such that $\left|(M / u)^{w}\right| \geq 2$. Assume on the contrary that for every non-loop element $u \in E$ we have $\left|(M / u)^{w}\right|=1$. Then every pair of bases $B_{1}, B_{2}$ of $M$ with $B_{1}^{w} \neq B_{2}^{w}$. satisfies $B_{1} \cap B_{2}=\emptyset$ otherwise for every $z \in B_{1} \cap B_{2}$, $\left|(M / z)^{w}\right| \geq 2$. Now, for every $z \in B_{1}$, there is $z^{\prime} \in B_{2}$ such that $C=\left(B_{1} \backslash\{z\}\right) \cup\left\{z^{\prime}\right\}$ is a base of M. For such a base $C, B_{1} \cap C \neq \emptyset, B_{2} \cap C \neq \emptyset$, and we must have $B_{1}^{w}=C^{w}=B_{2}^{w}$, a contradiction.

Applying the chain of inequalities proving (2) with $y=u$. We have

$$
\left|M^{w}\right|=\left|(M / u)^{w}+G_{u}\right|=\left|(M / u)^{w}\right|+\left|G_{u}\right|-1 .
$$

Note that $w(E \backslash\{u\}) \subset w(E)$, clearly verifies the Pollard condition. If $\left|G_{u}\right| \geq 2$ Theorem $\mathrm{A}$ implies that $M^{w}$ is a progression and thus $M$ satisfies Condition (i) of the theorem, contradicting our assumption on $M$. We must have $\left|G_{u}\right|=1$.

Thus $G_{u}=\{w(u)\}$ and $M^{w}=w(u)+(M / u)^{w}$.

Since the translate of a progression is a progression, $M / u$ is not a progression. By Lemma 2 , $(M / u)$ and $M / c l(u)$ have the same bases and thus the result holds if $r(M)=2$. If $r(M)>2$, then by the Induction hypothesis there is a hyperplane $H$ of $M / u$ such that $(M / u)^{w}=(M / u / H)^{w}=$ $\left(M /(C l(\{u\} \cup H))^{w}\right.$, and (ii) holds.

Corollary 3 (Vosper's Theorem [16, 17]) Let $p$ be a prime and let $A, B$ be subsets of $\mathbb{Z}_{p}$ such that $|A|,|B| \geq 2$.

$$
\text { If }|A+B|=|A|+|B|-1<p \text { then one of the following holds: }
$$

(i) $c-A=\left(\mathbb{Z}_{p} \backslash B\right)$.

(ii) $A$ and $B$ are arithmetic progressions with a same difference.

Proof. Consider the matroid $N=\left(\mathcal{U}_{1}(A) \oplus \mathcal{U}_{1}(B)\right)$ and its weight function $w$ defined in the Introduction. $H=A \times\{0\}$ and $H^{\prime}=B \times\{1\}$ are the hyperplanes of $N$ and we have $N^{w}=A+B$.

If $\left|N^{w}\right|=|A|+|B|-1$ then Theorem 1 says that $N$ must satisfy one of its conditions (i) or (ii). Since by hypothesis $|A|,|B| \geq 2$ we have $\left|N^{w}\right|>\max (|A|,|B|) \geq\left|(N / H)^{w}\right|,\left|\left(N / H^{\prime}\right)^{w}\right|$ and we conclude that $N^{w}$ must be an arithmetic progression with difference $d$. Without loss of generality we may take $d=1$. 
Case 1. $|A+B|=p-1$. Put $\{c\}=\mathbb{Z}_{p} \backslash(A+B)$. We have $c-A \subset\left(\mathbb{Z}_{p} \backslash B\right)$. Since these sets have the same cardinality we have $c-A=\left(\mathbb{Z}_{p} \backslash B\right)$.

Case 2. $|A+B|<p-1$.

We have $|A+B+\{0,1\}|=|A+B|+1=|A|+|B|<p$.

We must have $|A+\{0,1\}|=|A|+1$, since otherwise by the Cauchy-Davenport Theorem,

$$
\begin{aligned}
|A+B|+1 & =|A+B+\{0,1\}| \\
& =|A+\{0,1\}+B| \\
& \geq(|A|+2)+|B|-1=|A|+|B|+1,
\end{aligned}
$$

a contradiction. It follows that $A$ is an arithmetic progression with difference 1 . Similarly $B$ is an arithmetic progression with difference 1 .

\section{References}

[1] Caro, Yair Zero-sum problems, a survey. Discrete Math. 152 (1996), no. 1-3, 93-113.

[2] A. L. Cauchy, Recherches sur les nombres, J. Ecole Polytechnique 9 (1813), 99-116.

[3] I. Chowla, A theorem on the addition of residue classes: applications to the number $\Gamma(k)$ in Waring's problem, Proc.Indian Acad. Sc., Section A, no. 1 (1935) 242-243.

[4] H. Davenport, On the addition of residue classes, J. London Math. Soc. 10(1935), 30-32.

[5] J.A. Dias da Silva and M.B. Nathanson, "Maximal Sidon sets and matroids", Discrete Math. to appear.

[6] P. Erdős, A. Ginzburg and A. Ziv, A theorem in additive number theory, Bull Res. Council Israel $10 F(1961), 41-43$.

[7] A. Geroldinger, F. Halter-Koch, Non-unique factorizations. Algebraic, combinatorial and analytic theory. Pure and Applied Mathematics (Boca Raton), 278. Chapman \& Hall/CRC, Boca Raton, FL, 2006. xxii+700 pp.

[8] Y.O. Hamidoune, An isoperimetric method in additive theory. J. Algebra 179 (1996), no. $2,622-630$.

[9] J. H. B. Kemperman, On small sumsets in abeliangroups, Acta Math. 103 (1960), 66-88.

[10] M. B. Nathanson, Additive Number Theory. Inverse problems and the geometry of sumsets, Grad. Texts in Math. 165, Springer, 1996.

[11] J. M. Pollard, A generalisation of the theorem of Cauchy and Davenport, J. London Math. Soc. (2) 8 (1974), 460-462.

[12] J. M. Pollard, Addition properties of residue classes, J. London Math. Soc. (2) 11 (1975), no. $2,147-152$. 
[13] I. P. F. da Silva, Orientability of Cubes, Discrete Math. 308 (2008), 3574-3585.

[14] A. Schrijver, P.D. Seymour, Spanning trees of different weights. Polyhedral combinatorics (Morristown, NJ, 1989), 281-288, DIMACS Ser. Discrete Math. Theoret. Comput. Sci., 1, Amer. Math. Soc., Providence, RI, 1990.

[15] T. Tao and V.H. Vu, Additive Combinatorics, Cambridge Studies in Advanced Mathematics 105 (2006), Cambridge Press University.

[16] G. Vosper, The critical pairs of subsets of a group of prime order, J. London Math. Soc. 31 (1956), 200-205.

[17] G. Vosper, Addendum to "The critical pairs of subsets of a group of prime order", J. London Math. Soc. 31 (1956), 280-282.

[18] Welsh, D.J.A., Matroid Theory, Academic Press, London, 1976.

[19] White, N. (ed), Theory of Matroids, Cambridge University Press, 1986. 